DOI: 10.3901/JME.2021.03.227

\title{
复杂陶瓷型芯增材制造及浇注工艺验证"
}

\author{
胡可辉 ${ }^{1,2}$ 吕志刚 ${ }^{1,2}$ 陆 宽 ${ }^{3}$ 梁静静 $^{4}$ \\ (1. 清华大学摩擦学国家重点实验室 北京 100084; \\ 2. 清华大学机械工程系 北京 100084; \\ 3. 北京十维科技有限责任公司 北京 100092; \\ 4. 中国科学院沈阳金属材料研究所 沈阳 110016)
}

\begin{abstract}
摘要: 针对多层内腔空心浴轮叶片用复杂陶瓷型芯, 研究基于光固化的陶瓷型芯增材制造方法。采用光敏树脂与熔融石英粉 混制的陶瓷浆料, 通过 DLP 增材制造设备进行陶瓷型芯坏体制备, 再经过脱脂烧结工艺获得可用于实际浇注的陶瓷型芯。光 敏树脂活性单体选用 1,6-已二醇二丙烯酸酯(1,6-hexanediol diacrylate, HDDA)和乙氧化季戊四醇四丙烯酸酯(Ethoxylated pentaerythritol tetraacrylate, PPTTA), 熔融石英粉体采用 400 目和 1000 目的粉体进行级配。坏体分层制造中, 发现浆料铺料 厚度影响坏体受力, 通过调整加料方式、刮刀位置、刮料速度, 实现浆料厚度的准确控制。最终成功制备出高 $120 \mathrm{~mm}$ 、宽 $80 \mathrm{~mm}$ 的多层复杂结构陶瓷型芯, 并在实际工艺条件下进行金属浇注和脱芯验证, 力学性能和脱芯性能满足工艺要求。
\end{abstract}

关键词：陶瓷型芯；光固化；增材制造；熔模铸造；浴轮叶片

中图分类号: TG241

\section{Additive Manufacturing of Complex Ceramic Cores and Verification of Casting Process}

\author{
HU Kehui ${ }^{1,2}$ LÜ Zhigang ${ }^{1,2}$ LU Kuan ${ }^{3}$ LIANG Jingjing ${ }^{4}$
}

(1. State Key Laboratory of Tribology, Tsinghua University, Beijing 100084;

2. Department of Mechanical Engineering, Tsinghua University, Beijing 100084;

3. Beijing Ten Dimensions Technology Co., Ltd., Beijing 100092;

4. Institute of Metal Materials, Chinese Academy of Sciences, Shenyang 110016)

\begin{abstract}
Additive manufacturing technology based on SLA is investigated to produce multi-wall ceramic cores for large-size blades with complex hollow structure. The green part is prepared with DLP printer from ceramic slurry mixed of photoresin and fused silica powder. After debinding and sintering, the ceramic core using for real casting process is fabricated. The resin consisted of 1,6-hexanediol diacrylate(HDDA)and ethoxylated pentaerythritol tetraacrylate(PPTTA). The fused silica had two powder sizes, 400 mesh and 1000 mesh respectively. During the process of printing layer by layer, the stress state of the green part was found to be influenced by the thickness of ceramic coating, which could be optimized by adjusting coating process, blade gap and velocity. A multi-layer ceramic core( height: 120 $\mathrm{mm}$, width: $80 \mathrm{~mm}$ ) is eventually created and verified by real casting and core-removing process.
\end{abstract}

Key words: ceramic core; stereolithography; additive manufacturing; investment casting; gas turbine blade

\section{0 前言}

航空发动机和燃气轮机是航空、军工、能源等 产业的重要装备, 空心浴轮叶片作为装备的核心部

* 国家重点研发计划资助项目(2018YFB1106600)。20200211 收到初稿, 20201014 收到修改稿
件，被誉为 “制造业皇冠上的明珠” ${ }^{[1]}$ 。空心浴轮 叶片主要采用熔模铸造工艺生产 ${ }^{[2]}$ ，而陶瓷型芯是 形成空心叶片复杂内腔的关键 ${ }^{[3]}$ 。

为了提升航空发动机和燃气轮机的性能, 需要 提高叶片冷却效率以满足在更高温度下工作的需求, 因此叶片冷却道内腔结构设计日趋复杂，对陶瓷型 芯的形状复杂度、结构精细度不断提出更高要求 ${ }^{[4]}$ 。 而陶瓷型芯一般采用热压注法制备, 由于采用金 
属模具, 对所制备的陶瓷型芯复杂程度有限制。 增材制造具有易于成形复杂形状的优势, 研 究者不断尝试将增材制造与陶瓷铸型或型芯制备 相融合, 拓展铸造工艺方法。美国密歇根大学和 佐治亚理工大学采用大面积无掩膜光聚合技术 (Large area maskless photopolymerization, LAMP)制 备了尺寸约 $10 \mathrm{~mm}$ 的简单形状叶片陶瓷铸型 ${ }^{[5-6]}$, 2012 年公开报道铸造出空心叶片 ${ }^{[7]}$ 。华中科技大 学叶春团队 ${ }^{[8-9]}$ 利用 $3 \mathrm{D}$ 打印(3D printing, 3DP)技 术以纳米氧化锆县浮液作为黏结剂制备了钙基陶 瓷型芯, 并研究了其烧结性能。西安交通大学李 涤尘才队 ${ }^{[10-11]}$ 通过光固化增材制造技术打印树脂 模具, 再通过陶瓷浆料的灌注实现了一体化陶瓷 铸型的制备, 并浇注出金属零件。清华大学吕志 刚团队 ${ }^{[12-13]}$ 采用光固化陶瓷增材制造技术制备 熔模铸造型壳/型芯, 并对高精度成形原理进行了 揭示。

在陶瓷增材制造方法中, 基于光固化的陶瓷增 材制造工艺因其成形精度高, 具有实现高精度复杂 形状陶瓷型芯的工艺可行性。但对于大尺寸的陶瓷 型芯, 特别是多层壁型芯的制备, 需要系统优化工 艺, 并通过实际浇注验证增材制造陶瓷型芯的综合 性能。

\section{1 材料与方法}

\section{1 陶瓷型芯材料的选择}

陶瓷浆料由可光聚合的预混液和陶瓷粉体组成。 其中预混液包括活性单体、光引发剂、分散剂。活 性单体为 1,6-己二醇二丙烯酸酯 $(1,6$-hexanediol diacrylate, HDDA)和乙氧化季戊四醇四丙烯酸酯 (Ethoxylated pentaerythritol tetraacrylate, PPTTA); 光引发剂含量为单体质量的 $1 \%$ 分散剂添加量为粉 体质量的 $3 \%$ 。

硅基陶瓷型芯具有热膨胀系数小、抗热震性能 优良、退让性和溶出性好的优势 ${ }^{[14]}$, 采用高纯熔融 石英粉体作为制备复杂陶瓷型芯的基础骨料。为了 提高陶瓷浆料的流动性, 选择了 400 目和 1000 目 两种粉体按照 $1: 1$ 进行级配, 表 1 为熔融石英粉的 化学成分。

表 1 熔融石英化学成分(质量分数\%)

\begin{tabular}{ccccc}
\hline $\mathrm{SiO}_{2}$ & $\mathrm{Fe}_{2} \mathrm{O}_{3}$ & $\mathrm{MgO}$ & $\mathrm{Na}_{2} \mathrm{O}$ & $\mathrm{Al}_{2} \mathrm{O}_{3}$ \\
\hline$\geqslant 99$ & $\leqslant 0.1$ & $\leqslant 0.05$ & $\leqslant 0.05$ & $\leqslant 0.1$ \\
\hline
\end{tabular}

将陶瓷粉料和预混液混合, 搅拌均匀后球磨
$12 \mathrm{~h}$, 制备出固含量为 $55 \%$ (体积分数)的陶瓷浆料, 其黏度为 $4 \mathrm{~Pa} \cdot \mathrm{S}$ 。

\section{2 设备和成形特点}

采用数字光处理(Digital light process, DLP)光 固化陶瓷增材制造工艺 ${ }^{[15]}$, 设备为北京十维科技有 限责任公司的 AutoCera-M 型陶瓷打印机。

该设备的结构原理如图 1 所示。

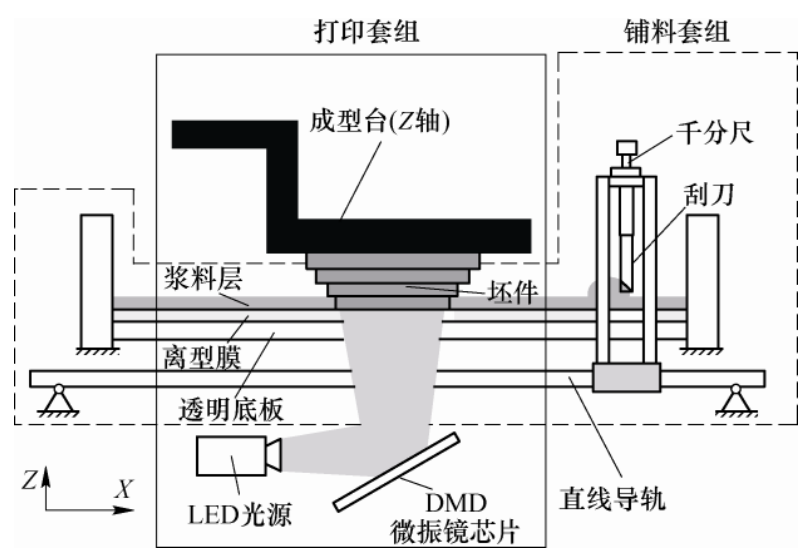

图 1 光固化陶瓷增材制造设备结构原理图

设备分为铺料组件和打印组件。虚线框内为铺 料组件，包括刮刀、千分尺、透明底板、离型膜、 直线导轨、浆料层。实线框内为打印组件, 包括 LED 光源、 DMD 微振镜芯片、成型台。

启动打印前, 导入三维数字模型, 将陶瓷浆料 倒入料盒中, 旋转千分尺手动调整刮刀到一定高度 $h_{\mathrm{b}}$, 设定好曝光参数(光机功率密度 $W$ 和单层曝光时 间 $t$ )和刮刀行进速度 $v_{\mathrm{b}}$, 然后开始打印。

开始打印后, 刮刀往复运动使浆料在料盒的离 型膜上均匀涂挂一层厚度为 $\delta$ 的浆料层, 成型台下 降到其底部与离型膜之间为一个切片厚度 $\delta_{0}$ 的间隙, 随后 LED 光源投影要固化的截面形状, 曝光区域的 陶瓷浆料固化。成型台提升, 然后再铺料, 重复下 一层曝光固化过程, 层层累积最后得到陶瓷零件的 坏体。

该设备为倒置式设计, 即光源位于成型台下方 向上投影。该方式的优点是用浆料少且不易沉降、 光学投影精度高, 但缺点是坏件上升时需要克服与 离型膜间的黏附力, 存在坏件断裂或掉落风险 ${ }^{[12,15]}$ 。

\section{3 工艺参数}

工艺参数主要包括坏件成形阶段的打印参数 和脱脂烧结阶段的温度制度，匹配适宜的工艺参 数才能实现型芯的完整成形及其精度、力学性能 要求。

打印参数如表 2 所示。 


\begin{tabular}{lc}
\hline \multicolumn{1}{c}{ 参数 } & 数值 \\
\hline 厚度 $\delta_{0} / \mu \mathrm{m}$ & 50 \\
高度 $h_{\mathrm{b}} / \mu \mathrm{m}$ & 100 \\
光机功率密度 $W /\left(\mathrm{mW} / \mathrm{cm}^{2}\right)$ & 30 \\
单层曝光时间 $t / \mathrm{s}$ & 10 \\
刮刀行进速度 $v_{\mathrm{b}} /(\mathrm{mm} / \mathrm{s})$ & 135 \\
\hline
\end{tabular}

脱脂烧结工艺温度制度如图 2 所示。

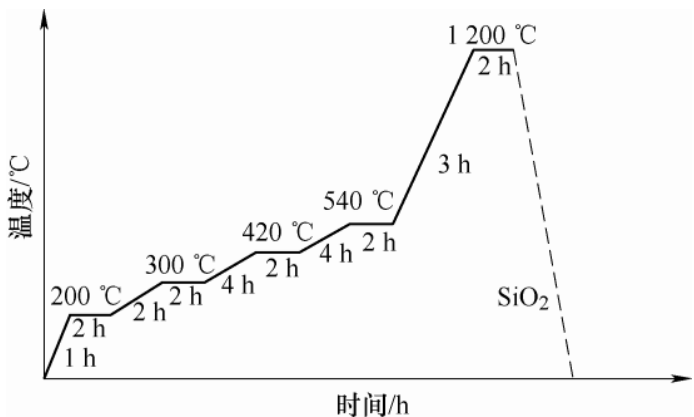

图 2 脱脂烧结温度制度

\section{4 性能表征方法}

\subsection{1 铺料厚度}

铺料厚度为刮刀运动后在离型膜表面涂覆的浆 料涂层厚度, 使用湿膜轮和湿膜梳进行测量。其中 湿膜轮的分辨率为 $2.5 \mu \mathrm{m}$, 量程为 $0 \sim 100 \mu \mathrm{m}$; 湿 膜梳的分辨率为 $10 \mu \mathrm{m}$, 量程为 $20 \sim 370 \mu \mathrm{m}$ 。

\subsection{2 显气孔率、吸水率、体积密度}

采用尺寸为 $\phi 4 \mathrm{~mm} \times 50 \mathrm{~mm}$ 的圆柱体样品, 烧结 后保证表面干净、平整, 外观无裂纹、气泡等缺陷。 应用阿基米德原理(排水法), 显气孔率按照式(1), 吸水率按照式(2), 体积密度按照式(3)计算。

$$
\begin{gathered}
B=\frac{m_{2}-m_{1}}{m_{2}-m_{3}} \times 100 \% \\
\rho_{\mathrm{r}}=\frac{m_{2}-m_{1}}{m_{1}} \times 100 \% \\
\rho_{\mathrm{v}}=\frac{m_{1} \times \rho_{\mathrm{s}}}{m_{2}-m_{3}} \times 100 \%
\end{gathered}
$$

式中, $B$ 为显气孔率; $\rho_{\mathrm{r}}$ 为相对致密度; $\rho_{\mathrm{v}}$ 为体积 密度; $m_{1}$ 为干燥试样质量; $m_{2}$ 为吸水饱和试样在空 气中的质量; $m_{3}$ 为吸水饱和试样在水中的质量; $\rho_{\mathrm{s}}$ 为试验温度下水的密度。

\subsection{3 烧成收缩率}

收缩率样品为 $5 \mathrm{~mm} \times 5 \mathrm{~mm} \times 5 \mathrm{~mm}$ 的正方体。 烧结收缩率用式(4)进行计算, 不同方向 $(x 、 y 、 z)$ 以 设备的坐标轴为准, 其中 $z$ 方向为固化层累积的 方向。

$$
S_{\mathrm{s}}=\frac{d_{\mathrm{g}}-d_{\mathrm{s}}}{d_{\mathrm{g}}} \times 100 \%
$$

式中, $S_{\mathrm{s}}$ 为烧结收缩率; $d_{\mathrm{s}}$ 为烧结后的尺寸; $d_{\mathrm{g}}$ 为 坏体尺寸。

\subsection{4 室温抗弯强度}

使用电子万能试验机对烧结后室温抗弯强度进 行测试。试样破坏时的载荷在测试设备上直接读出, 试样精确尺寸使用游标卡尺进行测量, 抗弯强度 $M$ 可使用式(5)进行计算。

$$
M=\frac{3 L S}{2 W_{\mathrm{s}} T^{2}}
$$

式中, $L$ 为型芯材料破坏载荷; $S$ 为支点跨距; $W$ s 为样条宽度; $T$ 为样条厚度。

由于增材制造试样有分层结构, 试样不同成形 方向的力学性能存在各向异性, 因此测量抗弯强度 需要定义测试方向。如图 3 所示, 分层面水平方向 为 $x-y$ 方向, 分层面坚直方向为 $z$ 方向即测试结果 为层间强度。样件尺寸为 $60 \mathrm{~mm} \times 10 \mathrm{~mm} \times 4 \mathrm{~mm}$, 跨 距为 $30 \mathrm{~mm}$ 。

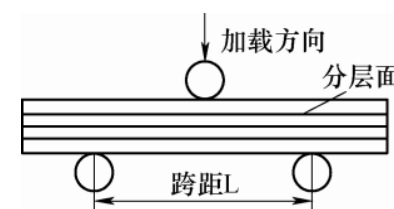

(a) $x-y$ 方向

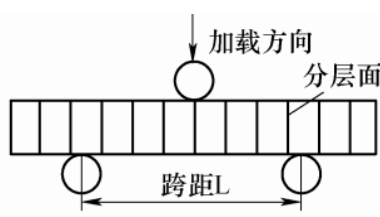

(b) $z$ 方向
图 3 加载方向与试样摆放形式

\subsection{5 热变形量}

采用双支点法测量, 如图 4 所示, 跨距为 $100 \mathrm{~mm}$, 样品尺寸为 $2 \mathrm{~mm} \times 6 \mathrm{~mm} \times 120 \mathrm{~mm}$ 。

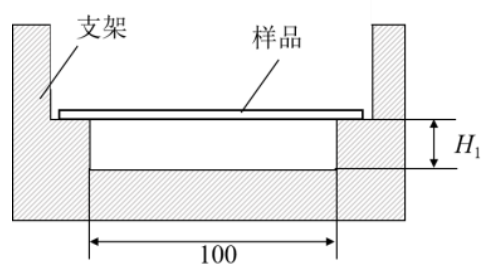

图 4 热变形量测量示意图

将试样支架放入加热炉中，升温至 $1500^{\circ} \mathrm{C}$ 保温 $30 \mathrm{~min}$ 。待炉温降至 $100^{\circ} \mathrm{C}$ 以下, 取出试样支架, 测 量试样最低点离支架底部的高度 $H_{2}$, 按照式(6)计算。

$$
\Delta H=H_{1}-H_{2}
$$

式中, $\Delta H$ 为热变形量; $H_{1}$ 为试样实验前高度; $H_{2}$ 为试样实验后高度。

\subsection{6 表面粗糙度}

使用台阶仪(美国, KLA Tencor P7)测试型芯烧 结后的表面粗粘度, 设备横向分辨率小于 $0.1 \mu \mathrm{m}$ 。 


\section{2 浆料铺料厚度对坏体受力的影响}

\section{1 陶瓷型芯的结构分析}

型芯打印时的分层方式会对制备过程中的坏体 受力产生重要影响, 曝光固化截面越大, 坏件打印 完一层后, 与分离膜的分离力越大, 容易产生缺陷 和掉落 ${ }^{[12]}$ 。图 $5 \mathrm{a}$ 为空心叶片模型图, 图 $5 \mathrm{~b}$ 为叶片 内腔对应陶瓷型芯的三维模型。根据该型芯结构特 征，采用坚直摆放打印以降低单层截面积。

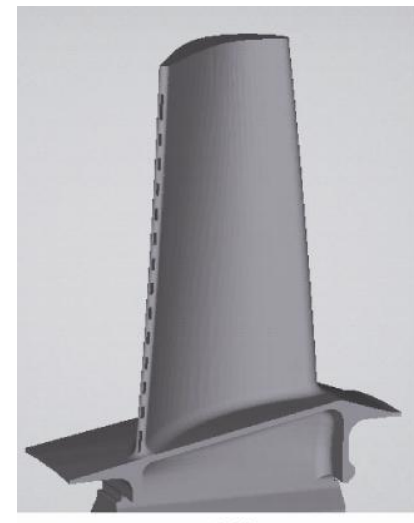

(a) 叶片模型

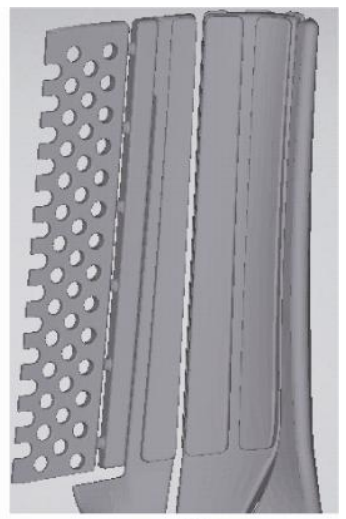

(b) 型芯模型
图 5 空心叶片及内腔对应型芯的三维数据模型

型芯为三层结构, 中间层为主体结构, 由三根 厚、宽为 5 10 mm、长约 $120 \mathrm{~mm}$ 的长柱及厚度为 1 2 mm 的带孔排气边构成; 前后两层分别为三条 宽 3 5 mm、厚 1 2 mm、长约 $100 \mathrm{~mm}$ 的薄壁长 板构成, 薄壁长板与主体结构由小圆柱连接。

图 6 为型芯坚直摆放时的纵向剖面图, 由于是 长的薄板结构, 在坏体下降压入浆料过程中的受力 对坏体质量也会产生影响。

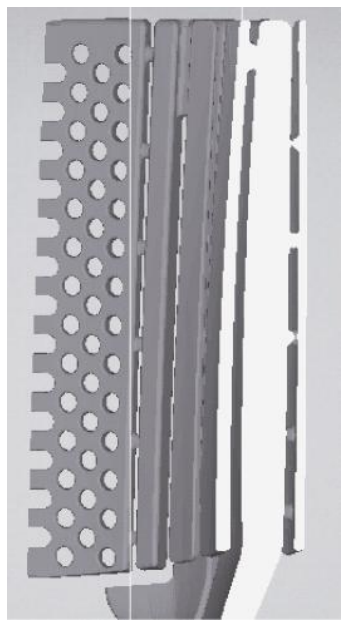

图 6 复杂型芯的坚直截面

\section{2 浆料层阻力模型}

假设打印一个半径为 $r_{0}$ 的圆柱，如图 7 所示。
在坏件单层成形时, 圆柱坏件以匀速 $v_{\mathrm{h}}$ 下降, 坏件 端面与浆料涂层接触后 (坏件端面高度为 $\delta$ )继续向 下运动至端面高度为 $\delta_{0}$ 的位置, 这个过程坏件会受 到浆料产生的反作用力。

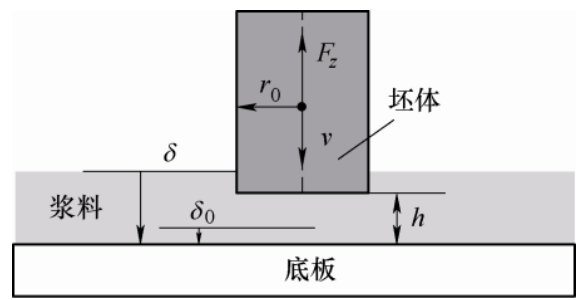

图 7 单层打印时坏体浸入浆料涂层示意图

黏性流体的运动规律可用纳维埃-斯托克斯 (Navier-Stokes)方程来描述。挤压黏性液膜对圆形上 表面的作用力可表示为 ${ }^{[16]}$

$$
F_{z}=-\frac{3 \pi \eta r_{0}^{4} v}{2 h^{3}}
$$

式中, $\eta$ 为浆料黏度; $h$ 为坏件端面离浆料涂层下表 面的高度。

由式(7)可知，随着坏件的下降，浆料层间隙越 来越小，坏件受到浆料的反作用力越来越大。当坏 件连续匀速向下运动, 陶瓷浆料无法及时完全从周 边间隙排出，坏件进一步受到浆料的反向挤压。

坏件下降过程中, 浆料反作用力所做的功由公 式(8)所示

$$
W_{F}=-\frac{3 \pi \eta r_{0}^{4} v}{2} \int_{\delta}^{\delta_{0}} \frac{1}{h^{3}} \mathrm{~d} h
$$

即

$$
W_{F_{z}}=\frac{3 \pi \eta r_{0}^{4} v}{4}\left(\frac{1}{\delta_{0}^{2}}-\frac{1}{\delta^{2}}\right)
$$

$F_{z}$ 做的功将一部分积累为坏件的形变势能, 由 式(9)可知, 坏件的形变与浆料黏度、下降速度、涂 层厚度相关, 如果确定浆料、打印模型、模型切片 厚度后, 铺料厚度 $\delta$ 是影响坏件变形的最关键因素。

\section{3 浆料层阻力造成的缺陷分析}

\subsection{1 错层缺陷}

当打印模型为薄壁长板结构或悬挑结构时, $\delta$ 的大小会影响坏件的变形程度。坏件在与离型膜形 成间隙的下压过程中缓慢浸入浆料，需要克服浆料 阻力 $F_{z}$, 其水平分力 $f_{1}$ 导致坏件产生变形。如图 $8 \mathrm{a}$ 所示, 虚线区域为坏件正常位置, 实线区域为坯件 在浆料作用力下变形后的实际位置, 新的一层开始 曝光后，在原正常位置形成新固化层，与已打印坏 件部分形成错层缺陷，该缺陷表现为坏件表面的层 
状横纹, 如图 $8 \mathrm{~b}$ 所示。 $\delta$ 越大, 浆料排出间隙的难 度就越大，造成的错层位移也就越大。

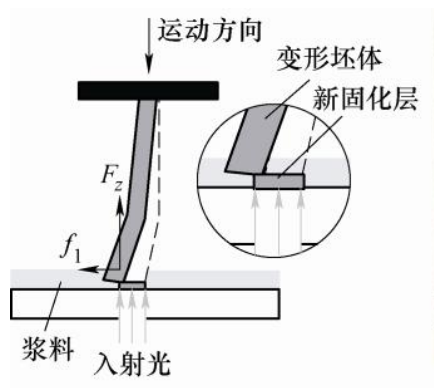

(a) 错层缺陷形成机制

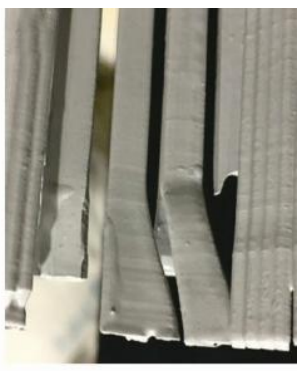

(b) 错层缺陷
图 8 错层缺陷示意图

\subsection{2 压溃缺陷}

当打印的结构截面较厚大时, 坏件在与离型膜 形成间隙的过程中浆料来不及从间隙中排出, 坏件 在 $F_{z}$ 的作用下被压缩, 与薄壁悬挑结构不同, 厚大 结构的弯曲变形能力有限, 坏件的变形方式主要是 压缩变形, 这导致模型底部和底板间的最终间隙大 于设定的切片厚度 $\delta_{0}$, 实际单层固化层厚与切片厚 度的误差为 $\varepsilon_{n}, n$ 层累计后, 坏体的实际高度为

$$
H=n \cdot \delta_{0}+\sum \varepsilon_{n}
$$

经过层厚增大效应的层层累加, 坏件所受压力 越来越大, 最终超过坏体的抗压极限强度而被压溃 造成坏件破坏, 即使坏件不被压溃也会积累很大内 应力导致尺寸误差和变形。 $\delta$ 越大, $\varepsilon_{n}$ 越大, 造成 的高度累计误差也会更大, 坏件更容易被压溃。 图 9 为被压溃缺陷示意图。

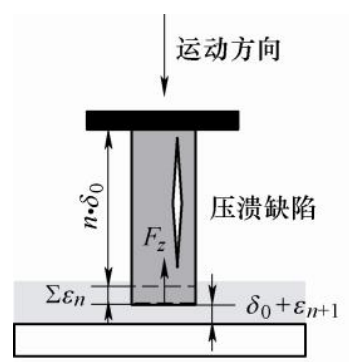

(a) 压溃缺陷形成机制

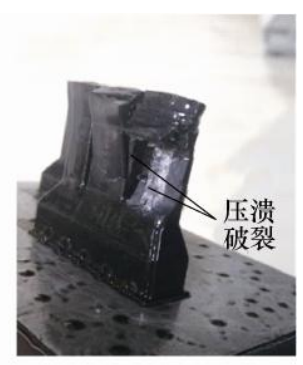

(b) 压溃缺陷
图 9 压溃缺陷示意图

\section{3 陶瓷浆料层厚度控制及坏体成形}

\section{1 陶瓷浆料层控制及其影响因素分析}

如图 10 所示, 刮刀高度为 $h_{\mathrm{b}}$, 内侧浆料的高度 为 $h_{\mathrm{s}}$, 刮刀以速度 $v_{\mathrm{b}}$ 运动, 获得浆料涂层的厚度为 $\delta$ 。

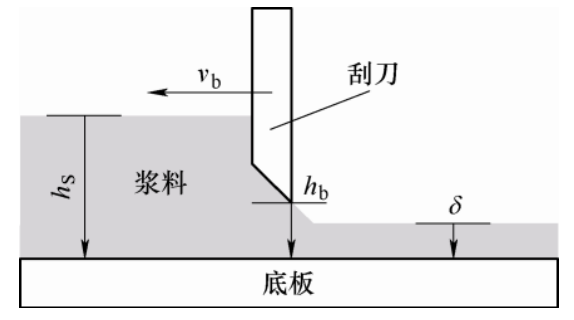

图 10 刮刀铺料状态示意图

图 11 为 $h_{\mathrm{b}}$ 一致时, 不同 $h_{\mathrm{s}}$ 对铺料状态影响示 意图。图 11a 为刮刀中储存大量浆料时的铺料状态, 图 11b 为采用喷头式加料器实时加料时的铺料状态, 分别对应 $h_{\mathrm{s}}$ 的高和低。当 $h_{\mathrm{s}}$ 较高时, 铺料过程更接 近传统流延成型系统中的流体流动过程 ${ }^{[17-18]}$ 。图 11a 中浆料在刮刀内侧产生压力 $P$, 导致刮刀运动时浆 料的相对 $11 \mathrm{~b}$ 流速更大; 图 $11 \mathrm{~b}$ 中除了浆料流速更 小之外, 刮刀运动时克服浆料黏滞性产生的阻力更 小, 因此能够形成更薄的浆料涂层。

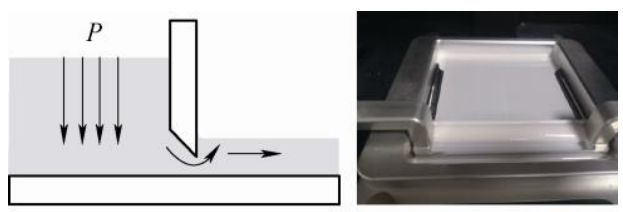

(a) 储料模式

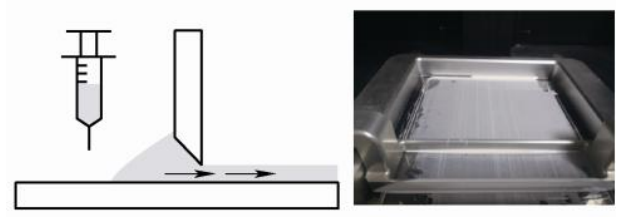

(b) 实时加料模式

图 11 铺料状态示意图

将刮刀紧贴料盒底 ( $h_{\mathrm{b}}$ 为极小值), 速度分别设 定为慢速 $(10 \mathrm{~mm} / \mathrm{s})$ 、中速 $(135 \mathrm{~mm} / \mathrm{s})$ 、快速 $(260 \mathrm{~mm} / \mathrm{s})$, 铺料后测量图 11 中两种情况的浆料涂 层厚度, 如图 12 所示。

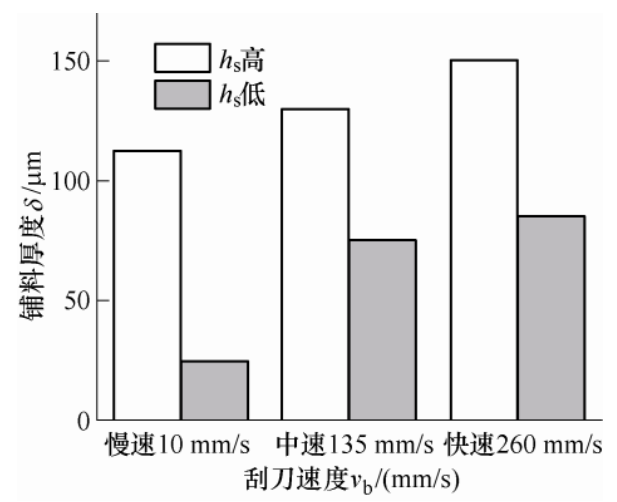

图 12 不同刮刀速度下浆料高度对铺料厚度的影响

可知此时得到的浆料涂层厚度为相应剪切速率 下可实现的极小值。 $h_{\mathrm{b}}$ 最小时, 刮刀和离型膜之间 
没有固定间隙, 浆料涂层产生的机理为“变形涂层”, 即刮刀在浆料的作用力下变形而在刮刀尖和底板间 产生间隙形成涂层。 $v_{\mathrm{b}}$ 越大, 刮刀与浆料之间的剪 切力越大, 刮刀变形产生的间隙越宽, 形成的 $\delta$ 更 厚。在图 $11 \mathrm{a}$ 所示条件下, $\delta$ 最小极限只能达到 $112.5 \mu \mathrm{m}$, 而图 $11 \mathrm{~b}$ 所示条件下可以获得最小极限 值 $25 \mu \mathrm{m}$ 。

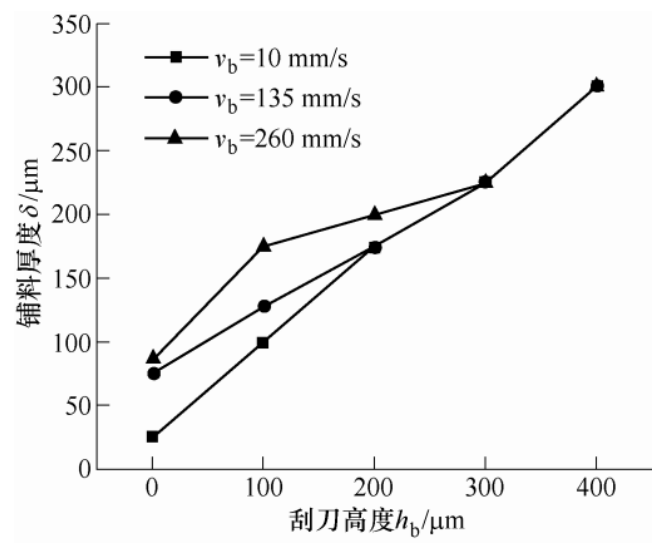

图 13 刮刀高度对铺料厚度的影响

刮刀高度对铺料厚度的影响如图 13 所示。慢、 中、快速三种条件下, 最小 $\delta$ 极限均在 $h_{\mathrm{b}}$ 为极小值 时获得, 分别为 $25 \mu \mathrm{m} 、 75 \mu \mathrm{m} 、 85 \mu \mathrm{m}$ 。当 $h_{\mathrm{b}}<100 \mu \mathrm{m}$, “变形涂层” 机制其主导作用, 所获得 $\delta>h_{\mathrm{b}}$, 且与 $v_{\mathrm{b}}$ 成正相关。当 $h_{\mathrm{b}}>200 \mu \mathrm{m}$, 所获得 $\delta<h_{\mathrm{b}}$, 此时 “间 隙涂层” 机制起主要作用, 即刮刀运动时由阻力导 致的变形减小, $\delta$ 主要由刮刀高度决定, 且慢速和 中速状态下已经没有差别。当 $h_{\mathrm{b}}>300 \mu \mathrm{m}, v_{\mathrm{b}}$ 不再 对 $\delta$ 产生影响。

在实际的打印过程中, $\delta>2 \delta_{0}$ 是保证正常打印的 必要条件 ${ }^{[12]}$ 。本陶瓷型芯的切片厚度 $\delta_{0}=50 \mu \mathrm{m}$, 选 择 $h_{\mathrm{b}}=100 \mu \mathrm{m}$ 及 $v_{\mathrm{b}}=135 \mathrm{~mm} / \mathrm{s}$, 此时 $\delta=125 \mu \mathrm{m}$ 。

\section{2 陶瓷型芯坏件成形及精度}

\section{2 .1 陶瓷型芯坏件成形}

执行表 2 的工艺参数, 型芯坏件可以实现与切 片厚度一致的 $50 \mu \mathrm{m}$ 均匀分层打印。图 14 为坏件断 面抛光分层结构电镜图, 断面可观察到均匀的分层 结构且层间结合面无明显缺陷。

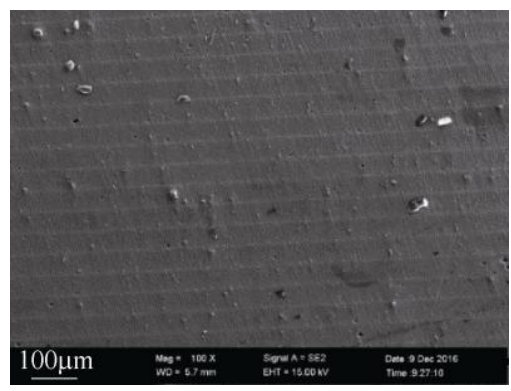

图 14 坏件断面分层结构示意图
图 15 为复杂型芯坏件成形过程的照片, 坏件完 整，表面光洁，没有产生错层和压溃缺陷。

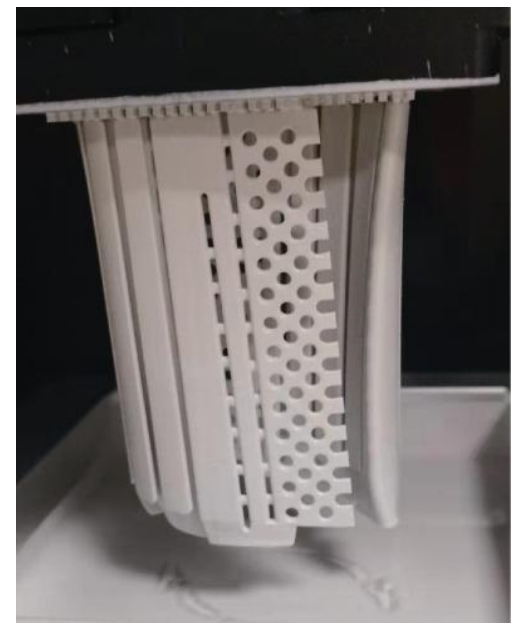

图 15 复杂型芯坏体的增材制造过程

\section{2 .2 坏件成形精度}

利用三维扫描仪对整个坏件进行了轮廓扫描, 并将扫描结果与 STL 数据模型进行对比, 如图 16 所示。坏体的尺寸偏差均低于 $0.2 \mathrm{~mm}$, 说明打印过 程中坏件受力均匀, 未产生明显的缺陷与变形。

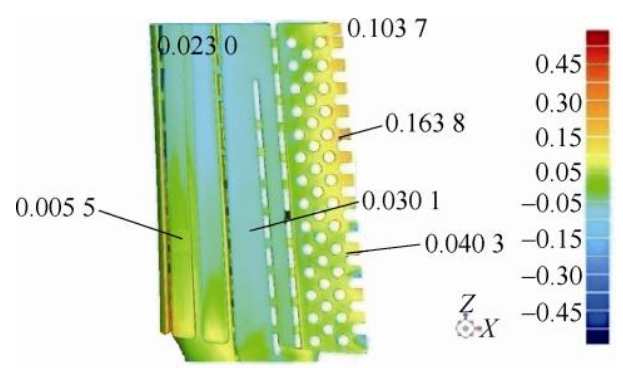

图 16 复杂陶瓷型芯坏件尺寸精度扫描对比图

\section{4 型芯性能和浇注验证}

\section{1 型芯性能}

型芯坏体经脱脂烧结后得到最终型芯, 利用第 1.4 节中的表征方法对型芯性能进行了检测, 得到表 3 。

表 3 型芯烧结后性能参数

\begin{tabular}{lc}
\multicolumn{1}{c}{ 参数 } & 数值 \\
\hline 室温抗弯强度 $/ \mathrm{MPa}$ & 18.4 \\
收缩率 $(\%)$ & $x-y: 4.2 ; z: 6$ \\
开孔隙率 $(\%)$ & 28.15 \\
吸水率 $(\%)$ & 17.26 \\
体积密度 $/\left(\mathrm{g} / \mathrm{cm}^{3}\right)$ & 1.67 \\
表面粗糙度 $/ \mu \mathrm{m}$ & 8.51 \\
高温挠度 $/ \mathrm{mm}$ & 7.48 \\
\hline
\end{tabular}




\section{2 浇注验证}

陶瓷型芯经过实际单晶铸造工艺条件下的浇铸 和脱芯验证, 获得了完整的单晶空心浴轮叶片样件。 浇铸时先焙烧型壳升至 $1550{ }^{\circ} \mathrm{C}$, 随后开始单晶 浇铸, 工作时长 $1 \mathrm{~h}$ 。图 $17 \mathrm{a}$ 为浇注用的型壳, 图 $17 \mathrm{~b}$ 为浇铸得到的叶片, 脱芯后内腔无型芯残留。 该实验验证了由本方法制备的硅基陶瓷型芯在强 度、脱除性、表面粗糙度方面均可满足工艺要求, 可铸造出无型芯残留、内腔表面质量优良的空心 叶片。

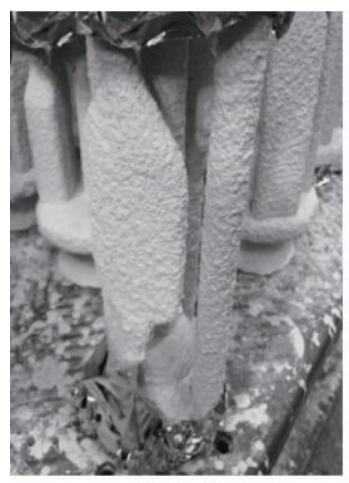

(a) 铸型

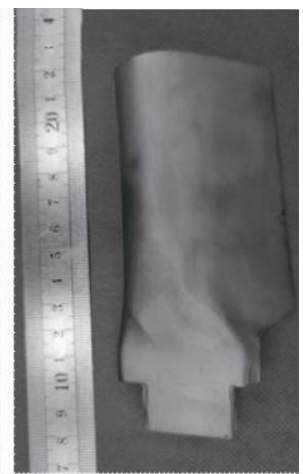

(b) 单晶叶片
图 17 空心叶片浇注验证

\section{5 结论}

（1）采用陶瓷光固化增材制造技术制备出高 $120 \mathrm{~mm}$ 的多层复杂陶瓷型芯, 并通过了包括单晶叶 片浇注和脱芯在内的全流程工艺验证, 证明了倒置 式 DLP 光固化增材制造技术在制备大尺寸复杂多 层陶瓷型芯具有现实可行性。

(2) 坏件所受压力过大造成错层缺陷和压溃缺 陷, 或导致坏件变形, 精确控制浆料涂层厚度, 减 少下压压力, 能够保证坏件精度。

(3) 浆料涂层厚度与加料形式、刮刀间隙高度、 刮刀速度有关, 通过控制这些参数可精确控制浆料 涂层厚度, 并实现 $25 \mu \mathrm{m}$ 薄浆料涂层, 为更高精度 坏件的成形打下基础。

\section{参 考 文 献}

[1] 赵效忠. 陶瓷型芯的制备和使用 $[\mathrm{M}]$. 北京: 科学出版 社, 2013.

ZHAO Xiaozhong. Preparation and application of ceramic core[M]. Beijing: Science Press, 2013.

[2] KALPAKJIAN S, SCHMID S. Manufacturing processes for engineering materials[J]. Journal of Applied Metalworking, 2004, 3(4): 446-446.
[3] 姜不居. 熔模精密铸造[M]. 北京: 机械工业出版社, 2004.

JIANG Buju. Investment casting[M]. Beijing: China Machine Press, 2004.

[4] 顾国红, 曹腊梅. 熔模铸造空心叶片用陶瓷型芯的发展 [J]. 铸造技术，2002，23(2): 81-83.

GU Guohong, CAO Lamei. Development of ceramic cores for investment casting hollow blades. [J]. Foundry Technology, 2002, 23(2): 81-83.

[5] BEA C J , HALLORAN J W . Integrally cored ceramic mold fabricated by ceramic stereolithography[J]. International Journal of Applied Ceramic Technology, 2011, 8(6): 1255-1262.

[6] HALLORAN J W , TOMECKOVA V , GENTRY S , et al. Photopolymerization of powder suspensions for shaping ceramics $[\mathrm{J}]$. Journal of the European Ceramic Society, 2011, 31(14): 2613-2619.

[7] DDM System. Our story [EB/OL].[2014-05-11]. http: // http: //www.ddmsys.com/our-story,

[8] ZHAO Huoping, YE Chunsheng, FAN Zitian. 3D printing of calcia-based ceramic core composites[J]. Advances in Science and Technology, 2014, 88: 65-69.

[9] ZHAO Huoping, YE Chunsheng, FAN Zitian, et al. 3D printing of $\mathrm{CaO}$-based ceramic core using nanozirconia suspension as a binder[J]. Journal of the European Ceramic Society, 2017, 37: 5119-5125..

[10] 赵碟, 李涤尘, 吴海华, 等. 基于光固化原型的燃气轮 机浴轮叶片整体式陶瓷铸型设计与制备 $[\mathrm{C}] / /$ 中国机械 工程学会.第五届全国快速成形与制造学术会议论文集. 2011: 67-73.

ZHAO Lei, LI Dichen, WU Haihua, et al. Design and fabrication of stereolithography-based integral ceramic molds for gas turbine blades[C]//Chinese Mechanical Engineering Society. Proceedings of the fifth national rapid prototyping and manufacturing conference, 2011: 67-73.

[11] 周伟召, 李涤尘, 周金南南, 等. 基于光固化的直接陶瓷 成形工艺[J]. 塑性工程学报, 2009，16(3)：204-207.

ZHOU Weizhao, LI Dichen, ZHOU Xinnan, et al. Direct fabrication process of ceramics based on stereolithography[J]. Journal of Plasticity Engineering, 2009, 16(3): 199-201.

[12] HU Kehui , WEI Yameng , LU Zhigang , et al. Design of a shaping system for stereolithography with high solid loading ceramic suspensions[J]. 3D Printing and Additive 
Manufacturing, 2018, 5(4): 311-318.

[13] LI Xinyi, HU Kehui, LU Zhigang. Effect of light attenuation on polymerization of ceramic suspensions for stereolithography[J]. Journal of the European Ceramic Society, 2019, 39(7): 2503-2509..

[14] MURATA A, KUME Y, HASHIMOTO F. Application of catastrophe theory to forced vibration of a diaphragm air spring[J]. Journal of Sound and Vibration, 1987, 112(1): $31-44$.

[15] SONG Xuan, CHEN Yong, LEE T, et al. Ceramic fabrication using mask-image-projection-based stereolitho-graphy integrated with tape-casting[J]. Journal of Manufacturing Processes, 2015(20): 456-464.

[16] DAVIS A, FRENKEL A. Cylindrical liquid bridges squeezed between parallel plates: Exact Stokes flow solutions and hydrodynamic forces[J]. Physics of Fluids A Fluid Dynamics, 1992, 4(6): 1105-1109.

[17] CHOU Ye. KO Ya. YAN Man. Fluid flow model for ceramic tape casting $[\mathrm{J}]$. Journal of the American Ceramic Society, 1987, 70(10): 280-282.

[18] TOK Alfred I Y, BOEY F Y C, LAM Y C. Non-Newtonian fluid flow model for ceramic tape casting[J]. Materials Science \& Engineering A, 2000, 280(2): 282-288.

作者简介: 胡可辉, 女, 1991 年出生, 博士, 助理研究员。主要研究方 向为陶瓷增材制造。

E-mail: hukehui@ tsinghua.edu.cn

吕志刚(通信作者), 男, 1972 年出生, 博士, 研究员, 博士研究生导师。 主要研究方向为熔模精密铸造、陶瓷增材制造。

E-mail: 1vzg@tsinghua.edu.cn 